\title{
Novas ocorrências de hepáticas (Marchantiophyta) para o estado do Pará, Brasil.
}

\author{
Ayumi OSAKADA ${ }^{1}$, Regina C. L. LISBOA²
}

\begin{abstract}
RESUMO
Durante o inventário das hepáticas da Serra dos Carajás, foram identificadas quatro novas ocorrências para o estado do Pará: Frullania beyrichiana (Lehm. \& Lindenb.) Lehm. \& Lindenb, Frullania kunzei (Lehm. \& Lindenb.) Lehm. \& Lindenb, Symphyogyna aspera Steph. e Lophocolea leptantha (Hook. \& Tayl.) Tayl. Esta última espécie, antes somente relacionada para as regiões Sudeste e Sul do Brasil, é nova ocorrência para o eixo Norte-Nordeste brasileiro. Cada espécie é acompanhada de comentários morfológicos, taxonômicos e ecológicos.
\end{abstract}

PALAVRAS-CHAVE

brioflora, Frullania spp, Lophocolea sp, Symphyogyna sp, Serra dos Carajás.

\section{New occurrences of liverworts for the Pará state, Brazil.}

\begin{abstract}
During the survey of liverworts from the "Serra dos Carajás", four species were identified as new occurrences for the Pará state: Frullania beyrichiana (Lehm. E Lindenb.) Lehm. E Lindenb, Frullania kunzei (Lehm. E Lindenb.) Lehm. E Lindenb, Symphyogyna aspera Steph. and Lophocolea leptantha (Hook. E Tayl.) Tayl. This last species was reported only for the South and Southeast regions of Brazil, being a new occurrence for the brazilian North-Northeast axle. Each species is followed by morfological, taxonomic and ecological commentaries.
\end{abstract}

KEY WORDS

bryoflora, Frullania $s p p$, Lophocolea $s p$, Symphyogyna $s p$, "Serra dos Carajás".

\section{INTRODUÇÃO}

Possuindo cerca de $150.000 \mathrm{Km}^{2}$, a Serra dos Carajás (5 $54^{\circ}-6^{\circ} 33^{\prime} \mathrm{S}$; $\left.49^{\circ} 53^{\prime}-50^{\circ} 34^{\prime} \mathrm{W}\right)$ é uma província mineral situada no sul do Pará. Segundo Silva (1991), caracteriza-se por uma série de serras descontínuas e morros afastada por imensos vales. Possui clima tropical, quente e úmido, com temperaturas entre 24,3 e $28,3^{\circ} \mathrm{C}$, sendo que nos platôs o clima é serrano, variando entre 21 e $23^{\circ} \mathrm{C}$.

Apresenta diversos ecossistemas como: floresta de terra firme, mata de várzea, capoeira e vegetação de canga (afloramento rochoso em jazida de ferro).

Lisboa (1994) indicou quatro espécies coletadas na Serra dos Carajás como novas ocorrências para o estado do Pará: Rhodobryum subverticillatum Broth., citado no trabalho como Bryum subverticillatum (Broth.) Ochi, Porotrichum substriatum (Hampe) Mitt., como P. plicatulum Mitt., Lepidopilum scabrisetum (Schwägr.) Steere e L. stolonaceum Müll. Hal. Segundo Churchill (1998), estas duas últimas espécies são consideradas sinônimos.
Posteriormente, Lisboa \& Ilkiu-Borges, A. (1997) referiram as espécies Campylopus pilifer Brid., como nova ocorrência para o estado do Pará, e Taxitbelium portoricense Williams, como primeira referência para o Brasil, ambas coletadas na Serra.

Moraes \& Lisboa (prelo) realizaram, recentemente, o inventário dos musgos da Serra dos Carajás, identificando vinte famílias distribuídas em oitenta e nove espécies e três variedades, das quais, Pbyllodon truncatulum (Müll. Hal.) W.R.Buck e Lepidopilum polytrichoides (Hedw.) Brid. são novas citações para o estado do Pará, e Leucomium steerei B.H.Allen \& Veling e Fissidens weirii Mitt. são novas ocorrências para a Amazônia brasileira.

Todos esses trabalhos citados referem-se ao grupo dos musgos, nenhum contendo qualquer estudo das hepáticas.

Este trabalho tem como objetivo, ampliar o conhecimento das briófitas no estado do Pará, com o estudo de novas ocorrências de hepáticas na Serra dos Carajás.

${ }^{1}$ Aluna de Agronomia da Universidade Federal Rural da Amazônia e Bolsista PIBIC/CNPq/MPEG. ayumiosakada@hotmail.com

${ }^{2}$ Pesquisadora Titular, MCT-Museu Paraense Emílio Goeldi, Dept. de Botânica. C.P. 399, CEP. 66040-170, Belém - Pará. regina@museu-goeldi.br 


\section{ACTA AMAZONICA}

NOVAS OCORRÊNCIAS DE HEPÁTICAS (MARCHANTIOPHYTA)

PARA O ESTADO DO PARÁ, BRASIL

\section{MATERIAL E MÉTODOS}

O material foi coletado no município de Parauapebas, nos anos de 1992 e 1993. As identificações foram realizadas utilizando-se chaves e descrição de autores: Hell (1969), Fulford (1976), Yano \& Mello (1999), Oliveira-e-Silva \& Yano (2000) e Michel (2001).

A comprovação de que as espécies ainda não haviam sido citadas para o estado do Pará foi determinada, utilizando-se "checklists" de Yano (1984, 1989 e 1995) e os trabalhos mais recentes já mencionados.

Todo material identificado será incorporado ao Herbário João Murça Pires, do Museu Paraense Emílio Goeldi (Herbário MG).

\section{RESULTADOS}

Foram identificadas três espécies de hepáticas, configuradas como novas citações para o estado do Pará e uma primeira referência para o eixo Norte-Nordeste do Brasil. Essas espécies são relacionadas a seguir, em ordem alfabética de família, sendo apresentado dados sobre o material examinado, comentários taxonômicos, morfológicos e ecológicos e distribuição geográfica, para cada uma.

\section{Jubulaceae}

Frullania beyrichiana (Lehm. \& Lindenb.) Lehm. \& Lindenb., Syn. Hep.: 460. 1845.

Basiônimo: Jungermannia beyrichiana Lehm. \& Lindenb. in Lehmann, Pug. Plant. 5:25. 1833.

Tipo: Brasil, Rio de Janeiro, Serra dos Órgãos, col. Beyrich (W).

Material examinado: Brasil, estado do Pará, município de Parauapebas, Serra dos Carajás, Serra Norte, mata ao redor da mina do igarapé Bahia, sobre pau seco, J. Sales $E$ C. Rosário 1056, 2.IV.1993, det. A. Osakada.

Comentários: possui gametófito de coloração castanho-avermelhada, filídios subimbricados com ápice apiculado, às vezes agudo, margens lisas, células da base irregulares com trigônios e espessamentos intermediários; estilete filiforme, geralmente com 4-5 células de comprimento. Lóbulos três vezes mais longos que largos, subparalelos e contíguos ao caulídio, cilíndricos e inflados. Anfigastros subovalados ou subretangulares, margens lisas, base auriculada, bífidos até $1 / 4$ de seu comprimento. Descrição mais detalhada em Michel (2001). A espécie foi coletada em beira de mata úmida, em ambiente onde a luminosidade é maior, associada à Cheilolejeunea rigidula (Mont.) R.M. Schust. e Lejeunea laetevirens Nees \& Mont.

Distribuição geográfica: segundo Stotler (1970), ocorre no México e na América Central. No Brasil, é referida para Bahia (Bastos et al., 2000), Minas Gerais (Ångstroem, 1876), Rio Grande do Sul, Acre (Lemos-Michel, 1980), Rio de Janeiro (Gottsche et al., 1844), São Paulo (Herzog, 1925) e Pernambuco (Pôrto, 1989, 1990).

Frullania kunzei (Lehm. \& Lindenb.) Lehm. \& Lindenb., Syn. Hep. 449. 1845.

Tipo: Cuba, West Indies.

Material examinado: Brasil, estado do Pará, município de Parauapebas, Serra dos Carajás, Serra Vermelha, Cedere II, V. II, na subida da Serra, sobre galho podre, J. Sales \& C. Rosário 718, 30.III.1993; idem, Serra Norte, na canga do N1, margem esquerda da estrada, mata da Mimosa, sobre galho verde, J. Sales E C. Rosário 792, 31.III.1993, det. A. Osakada.

Comentários: possui gametófito prostrado e bem aderido ao substrato. Filídios imbricados, lobos subarredondados, margens lisas, ápice obtuso, células subretangulares a alongadas, paredes geralmente espessas e com trigônios. Lóbulos obovalado-clavados, cerca de duas vezes mais longos que largos, inflados, subparalelos ao caulídio, comumente com a porção superior mais próxima ao caulídio que a base; estilete filiforme com 3-4 células de comprimento. Anfigastros ovalado-retangulares, bífidos até $1 / 3$ de seu comprimento. Descrição mais detalhada em Michel (2001). Ocorreu associada a Cheilolejeunea rigidula (Mont.) R.M. Schust., Mastigolejeunea auriculata (Wilson) Schiffn. e Lejeunea flava (Sw.) Nees.

Distribuição geográfica: apresenta distribuição neotropical. No Brasil, é citada para Amazonas (Griffin III, 1979), Ceará, Espírito Santo, Minas Gerais, Mato Grosso, Rio Grande do Sul, Sergipe, São Paulo (Yano, 1984, 1989, 1995), Pernambuco (Pôrto, 1990), Rio de Janeiro (Yano \& Costa, 1992), Acre (Vital \& Visnadi, 1994), Bahia (Vilas BôasBastos \& Bastos, 1998), Paraíba (Yano, 1993), Paraná (Yano \& Colletes, 2000) e Roraima (Santiago, 1997).

\section{Lophocoleaceae}

Lophocolea leptantha (Hook. \& Tayl.) Tayl., Syn. Hep. 694. 1847.

Basiônimo: Jungermannia leptantha Hooker f. \& Taylor, London Jour. Bot. 3:471. 1844.

Tipo: Cape Horn, col.J.D. Hooker

Material examinado: Brasil, estado do Pará, município de Parauapebas, Serra dos Carajás, Serra Norte, margem direita da cachoeira do rio Bahia, em ecossistema de várzea, sobre pedra, J. Sales \& C. Rosário 1006, 1.IV.1993, det. A. Osakada.

Comentários: apresenta filídios espalhados, mais ou menos ascendente, subretangular a ovalado-truncado, constrito abaixo do ápice bífido, sinus em geral lunulado, segmentos com ponta longa e unisseriada, células com paredes finas, trigônios pouco desenvolvidos. Anfigastros conados com filídios em ambos os lados, bífidos até $2 / 3$ do seu comprimento, com segmentos longos e finos, o dente lateral é longo, divergente (quadrífido). Descrição mais detalhada em Fulford (1976). A espécie encontrava-se 
associada à Archilejeunea parviflora (Nees) Schiffner, Prionolejeunea microdonta (Gottsche) Steph., Lejeunea laetevirens Nees \& Mont.

Distribuição geográfica: ocorre na Argentina, Chile, Ilhas Malvinas e Oceania. No Brasil, é reportada para Minas Gerais (Ångstroem, 1876), Rio Grande do Sul (Fulford, 1976) e São Paulo (Schiffner \& Arnell, 1964). Primeiro registro para o Norte-Nordeste brasileiro.

\section{Pallaviciniaceae}

Symphyogyna aspera Steph., Bot. Gaz. 58:403. 1914.

Tipo: México, Xalapa \& Texolo, col. W.I.G. Land $\varepsilon$ Charles R. Barnes (G 9897).

Material examinado: Brasil, estado do Pará, município de Parauapebas, Serra dos Carajás, Serra Norte, $\mathrm{Km}$ 42, em capoeira, no picadão a NW da margem direita do rio Águas Claras, na areia do rio, J. Sales \& C. Rosário 1167, 3.IV.1993; idem, sobre galho vivo, J. Sales \& C. Rosário 1168, 3.IV.1993, det. A. Osakada.

Comentários: possui gametófito de coloração verdeclara a verde-alaranjada, desenvolvendo-se prostrada, com a região apical ligeiramente ascendente. Talo ondulado a sinuoso, ramificado, lobado; bordo inteiro a irregularmente partido e de ápice obtuso. Nas bordas das asas, principalmente perto do ápice, encontram-se papilas mucilaginíferas marginais. Descrição mais detalhada em Hell (1969). A espécie ocorreu tanto isolada como associada à Cheilolejeunea sp, Rectolejeunea berteroana (Gottsche) A. Evans, Lejeunea caespitosa Lindenb., Aphanolejeunea sp, Plagiochila rutilans Lindenb. e $P$. raddiana Lindenb.

Distribuição geográfica: ocorre na Argentina, Bolívia, Colômbia, Cuba, El Salvador, Equador, Martinica, México, Paraguai e Porto Rico. No Brasil, é encontrada em Amazonas, Minas Gerais, Rio de Janeiro, Rio Grande do Sul (Evans, 1925), Espírito Santo (Schäfer-Verwimp, 1991), Pernambuco (Sá \& Pôrto, 1996), Santa Catarina (Hässel de Menéndez, 1961) e São Paulo (Herzog, 1927).

\section{DISCUSSÃO}

As quatro espécies relacionadas como novas para o estado do Pará, confirmam Lisboa (1994), que ressaltou que à medida que fossem feitas coletas e identificações, a diversidade específica das briófitas aumentaria.

Foi observado que Lophocolea leptantha era referida apenas para áreas de baixas temperaturas. Segundo Lisboa (1994), a Serra dos Carajás também apresenta temperaturas amenas, podendo-se imaginar que esta espécie seja de lugares de maiores altitudes, pouco presentes na Amazônia.

No Brasil, Symphyogyna aspera e Frullania beyrichiana eram citadas apenas para um Estado em toda região Norte. Já $F$. kunzei apresenta ampla distribuição na América tropical.
Como foi analisada apenas uma pequena parte do total de amostras coletadas na área, com a continuidade do estudo, o número de novas ocorrências pode aumentar.

\section{AGRADECIMENTOS}

À Companhia Vale do Rio Doce, a Fundação Margareth Mee, ao Fundo Nacional de Meio Ambiente (FNMA) e ao Conselho Nacional de Desenvolvimento Científico e Tecnológico (CNPq), pelo apoio financeiro dado ao projeto. Ao Hugo Jorge Santos pelo auxílio na elaboração do "Abstract".

\section{BIBLIOGRAFIA CITADA}

Ångstroem, J. 1876. Primae linae muscorum cognoscendorum, qui ad Caldas Brasilia sunt collecti. II. Hepaticae. Öfvers $K$. vetenskAkad. Förh. 33: 77-92.

Bastos, C.J.P., Yano, O.; Bôas-Bastos, S.B. 2000. Briófitas de campos rupestres da Chapada Diamantina, Estado da Bahia, Brasil. Revta brasil. Bot. 23(4): 357-368.

Churchill, S.P. 1998. Catalog of Amazonian Mosses. Nichinan, J. Hattori Bot. Lab. 85: 191-238.

Evans, A.W. 1925. The Lobate species of Symphyogyna. Trans. connect. Acad. Art E Science 27: 1-50.

Fulford, M.H. 1976. Manual of the leafy Hepaticae of Latin America IV. Mem. New York Bot. Gard. 11: 393-53.

Gottsche, C.M., Lindenberg, J.B.W.; Nees von Esenbeck, C.G.D. 1844. Syn. Hep. Hamburg, J.A. Meissner, 835p.

Griffin III, D. 1979. Guia Preliminar para as briófitas freqüentes em Manaus e adjacências. Acta Amazonica 9 (supl.): 1-67.

Hässel de Menéndez, G.G. 1961. Las espécies Argentinas del género Symphyogyna. Boletín de la Sociedad Argentina de Botânica 9: 233-260.

Hell, K.G. 1969. Briófitas talosas dos arredores da cidade de São Paulo. Bol. Fac. Filos. Univ. São Paulo 335, Bot. 25: 1-190.

Herzog, Th. 1925. Contribuições ao conhecimento da flora briológica do Brasil. Arch. Bot. Estado São Paulo I: 27-105.

Herzog, Th. 1927. Zwei Bryophyten Sammlungen aus Südamerika. Hedwigia 71: 332-350.

Lemos-Michel, E. 1980. O gênero Frullania (Hepaticopsida) no Rio Grande do Sul, Brasil. Dissertação de Mestrado. Universidade Federal do Rio Grande do Sul, Porto Alegre. 149p.

Lisboa, R.C.L. 1994. Adições à brioflora do Estado do Pará. Bol. Mus. Para. Emílio Goeldi, sér. Bot., 10(1): 15-42.

Lisboa, R.C.L. \& Ilkiu-Borges, A.L. 1997. Novas ocorrências de Bryophyta (musgos) para o estado do Pará, Brasil. Acta Amazonica 27(2): 81-102.

Michel, E.L. 2001. Hepáticas epifíticas sobre o pinbeiro-brasileiro no Rio Grande do Sul. Porto Alegre: Ed. Universidade Federal do Rio Grande do Sul. 191p.

Moraes, E.N.R.; Lisboa, R.C.L. prelo. Inventário dos musgos (Bryophyta) da Serra dos Carajás, Estado do Pará. Bol. Mus. Para. Emílio Goeldi, sér. Bot. 


\section{ACTA AMAZONICA}

NOVAS OCORRÊNCIAS DE HEPÁTICAS (MARCHANTIOPHYTA) PARA O ESTADO DO PARÁ, BRASIL
Oliveira-e-Silva, M.I.M.N.; Yano, O. 2000. Anthocerophyta e Hepatophyta de Mangaratiba e Angra dos Reis, Rio de Janeiro, Brasil. Boletim do Instituto de Botânica 13: 1-102.

Pôrto, K.C. 1989. Analyse floristique et de la bryoflore d'une forêt de plaine et d'une forêt d'altitude moyenne dans l'état de Pernambuco (Brésil). Tese de Doutorado. Uni. Paris XII, Val de Marne. 234p.

Pôrto, K.C. 1990. Bryoflores d'une forêt de plaine et d'altitude moyenne dans l'état de Pernambuco (Brésil): Analyse floristique. Cryptogam., Bryol. Lichénol. 11: 109-161.

Sá, P.S.A. \& Pôrto, K.C. 1996. Novos registros de Hepaticopsida (Bryophyta) para Pernambuco. Revista Nordestina de Biologia 11(1): 37-43.

Santiago, R.L. 1997. Estudos brioflorísticos de três formações vegetais no município de Bonfim-Roraima. Dissertação de Mestrado. Recife, Universidade Federal de Pernambuco. 124p.

Schäfer-Verwimp, A. 1991. Contribution to the knowledge of the bryophyte flora of Espírito Santo, Brazil. J. Hattori Bot. Lab. 69: 147-170.

Schiffner, V.; Arnell, S. 1964. Ergebnisse der botanischen Expedidition der kaiserlichen Akademie der Wissenschaften nach Südbrasilien 1901. II. Hepaticae. ...sterr. Akad. Wiss., Math.-Naturwiss. Al., Denkschr. 111: 1-156.

Silva, M.F.F. 1991. Análise florística da vegetação que ocorre sobre canga hematítica em Carajás, Pará (Brasil). Bol. Mus. Para. Emílio Goeldi, sér. Bot., 7(1): 79-107.

Stotler, R.E. 1970. The genus Frullania subgenus Frullania in Latin America. Nova Hedwigia 18: 397-555.
Vilas Bôas-Bastos, S.B. \& Bastos, C.J.P. 1998. Briófitas de uma área de cerrado no município de Alagoinhas, Bahia, Brasil. Tropical Bryology 15: 101-110.

Vital, D.M.; Visnadi, S.R. 1994. Bryophytes of Rio Branco Municipality Acre, Brazil. Tropical Bryology 9: 69-74.

Yano, O. 1984. Checklist of Brazilian liverworts and hornworts. J. Hattori Bot. Lab. 56: 481-548.

Yano, O. 1989. An additional checklist of Brazilian bryophytes. J. Hattori Bot. Lab. 66: 371-434.

Yano, O. 1993. Briófitas do Nordeste Brasileiro: estado da Paraíba, Brasil. Biologica Brasilica 5 (1-2): 87-100.

Yano, O. 1995. A new additional annotated checklist of Brazilian bryophytes. J. Hattori Bot. Lab. 78: 137-182.

Yano, O.; Costa, D.P. 1992. Novas ocorrências de briófitas no Brasil. Anais do 81 Congresso da Sociedade Botânica de São Paulo. São Paulo. p. 33-45.

Yano, O.; Mello, Z.R. 1999. Frullaniaceae dos manguezais do litoral sul de São Paulo, Brasil. Iheringia, sér. Bot. 52: 65-87.

Yano, O.; Colletes, A.G. 2000. Briófitas do Parque Nacional de Sete Quedas, Guaíra, PR, Brasil. Acta Bot. Bras. 14(2): 215-242. 